\title{
Morphological transformation of NGC 205?
}

\author{
Ivo Saviane ${ }^{1}$, Lorenzo Monaco ${ }^{2} \&$ Tony Hallas ${ }^{3}$ \\ ${ }^{1}$ European Southern Observatory, Chile \\ email: isaviane@eso.org \\ ${ }^{2}$ Dept. of Astronomy, University of Concepcion, Chile \\ ${ }^{3}$ Astrophoto, USA
}

\section{A dE galaxy with recent central star formation}

NGC 205 is a small galaxy $\left(M / M_{\odot}=0.7 \times 10^{9} ; M_{V}=-16.6\right)$ currently located $36^{\prime} \mathrm{NW}$ of M31. It is classified as dE because in ground-based images it appears as an elliptical body. However past investigations have revealed characteristics that are more typical of a disk galaxy: the specific frequency of globular clusters is 1.8; the large scale dynamics shows partial rotational support; there is a significant amount $\left(10^{6} M_{\odot}\right)$ of rotating gas (molecular and atomic) and dust; the central regions harbor a fairly complex stellar population, including a 100-500 Myr old nucleus surrounded by 50- and 100-Myr old stellar associations (see references in Monaco et al. 2009; M09). Very recently, thanks to HST/ACS imaging we have been able to reveal a young central 'field' population (M09), extending out to $\sim 40^{\prime \prime}$ in radius $(\sim 160 \mathrm{pc})$. The luminosity function of the main sequence can be fitted with Saviane et al. (2004) model of continuous star formation (SF) from at least $\sim 600 \mathrm{Myr}$ ago to $\sim 60 \mathrm{Myr}$ ago. We found that $1.5 \times 10^{5} M_{\odot}$ in stars were produced from $\sim 300$ Myr to $\sim 60$ Myr ago, with a SF rate of $7 \times 10^{-4} M_{\odot} \mathrm{yr}^{-1}$. A continuous SF seems to support the latest simulations of NGC 205 orbit: Howley et al. (2008) found that the galaxy must be moving with a velocity $300-500 \mathrm{~km} \mathrm{~s}^{-1}$ (comparable to the escape velocity) along an almost radial orbit, and it should be approaching M31 for the first time. An episodic SF triggered by passages through M31 disk every 300 Myr in a bound orbit (Cepa \& Beckman 1988) is excluded by our data.

\section{Substructures in and near the body of NGC 205}

Kormendy and collaborators have proposed that all $\mathrm{dE}$ galaxies were originally dwarf disks (e.g., Kormendy et al. 2009), so the results discussed above can be placed in the broader context of $\mathrm{dE}$ formation. In particular, Lisker et al. (2006) discovered a number of blue-center dEs in the Virgo cluster, and considered NGC 205 a local representative of this new $\mathrm{dE}(\mathrm{bc})$ class (see also Koleva et al. 2009). In Virgo, $\mathrm{dE}(\mathrm{bc})$ galaxies present flattening distributions indicating disk shapes and show no central clustering, which leads the authors to suggest galaxy harassment (Moore et al. 1996) as their likely formation mechanism. Harassment requires repeated encounters with large galaxies, so it should not work in a low-density group environment. It is therefore suggested that 'tidal stirring' (Mayer et al. 2001) could lead to morphological transformation in the case of NGC 205. An alternative mechanism is that of 'galaxy threshing' (Bekki et al. 2001). In the course of transformation gas is funneled to the center and forms a density excess which could explain the blue centers (Moore et al. 1998; Bekki et al. 2001; Mayer et al. 2001). The rise of SF in the center of NGC 205 in correspondence to a decline in the external regions (Koleva 2009) might be a signature of this phenomenon. If the $\mathrm{dE}$ is at its first encounter with M31, we might be seeing it at the earliest phases of this process, when 

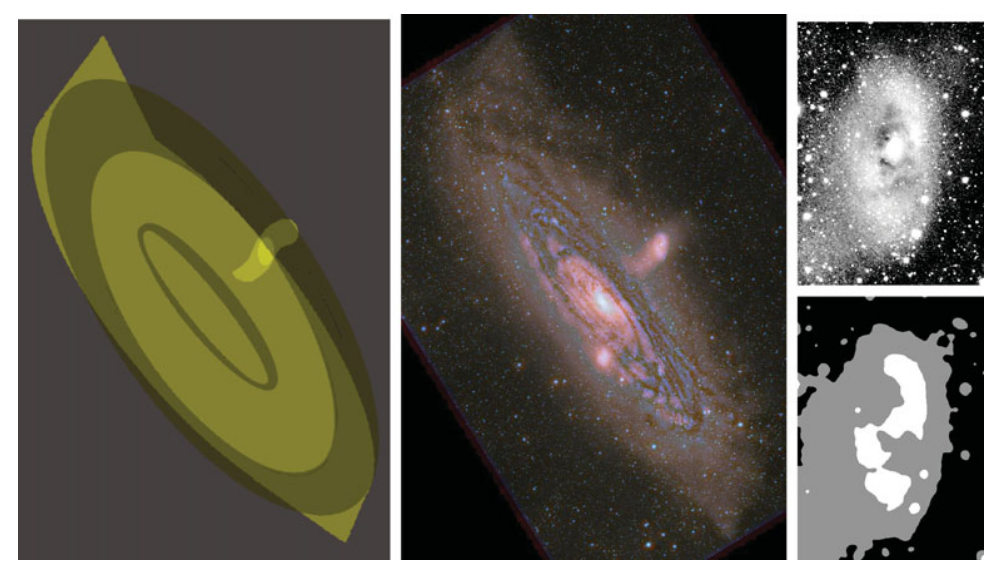

Figure 1. The central image of M31 and its satellites is based on a 9-frame mosaic taken with a SBIG/STL-11K camera and no filter, attached to a 6 -inch $\mathrm{f} / 8$ Stellarvue refractor. The red channel has been obtained by Gaussian smoothing with an $80 \mathrm{px}$ window, while the blue channel is the result of a four-pass unsharp-masking with a $50 \mathrm{px}$ window. The green channel is the original frame. The left panel is a schematic representation of the distribution of stars and dust in M31, screening NGC 205 and its tidal tails. The right panels show the unsharp masked image of NGC 205 (top) and a three-level contour version of the same frame (bottom).

still retaining most of its disk features. In the harassment scenario, bar and spiral features can be created and retained for some time. Indeed Lisker et al. (2006) present the case of VCC 0135 and VCC 1437 as showing possible spiral arms and a bar. In Fig. 1 we show that some sub-structure is also present inside the body of NGC 205, thanks to a two hour exposure frame, at a scale of $\sim 2^{\prime \prime} \mathrm{px}^{-1}$. The southern tidal tail stands out in a very prominent way, out to at least $17^{\prime}(\sim 3.5 \mathrm{kpc})$ from the galaxy center. The tail is probably longer, but it is hidden behind the high-extinction central regions of M31. The near absence of a northern tail might be explained by a very extended M31 dust ring screening it. This is plausible because NGC 205 is farther than M31 and the nearside of M31 is the NW one (see the schematic sketch in Fig. 1). Figure 1 further shows that the well-known dust clumps of NGC 205 seem to be part of a ring surrounding the central nucleus. Moreover, sub-structure is seen also across the main body of the galaxy, which resembles spiral arms connected by a bar. While this feature is quite intriguing in the context of morphological transformation, a foreground dust filament might also be responsible for the illusion. Very deep and wide-field imaging in three bands, such as that offered by the HST/ACS, would be needed to discriminate between the two hypotheses.

\section{References}

Bekki, K., Couch, W. J., Drinkwater, M. J., \& Gregg, M. D. 2001, ApJ, 557, L39

Cepa, J. \& Beckman, J. E. 1988 A\&A, 200, 21

Howley, K. M. et al. 2008, ApJ, 683, 722

Koleva, M. 2009, Ph.D. Thesis, Université Lyon I

Koleva, M. et al. 2009, MNRAS, 396, 2133

Kormendy, J., Fisher, D. B., Cornell, M. E., \& Bender, R. 2009, ApJS, 182, 216

Lisker, T., Glatt, K., Westera, P., \& Grebel, E. K. 2006, AJ, 132, 2432

Mayer, L. et al. 2001, ApJ, 559, 754

Monaco, L. et al. 2009, A\&A, 502, L9 (M09)

Moore, B., Katz, N., Lake, G., Dressler, A., \& Oemler, A. 1996, Nature, 379, 613

Moore, B., Lake, G., \& Katz, N. 1998, ApJ, 495, 139

Saviane, I., Hibbard, J. E., \& Rich, R. M. 2004, AJ, 127, 660 